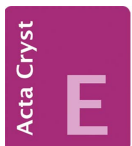

CRYSTALLOGRAPHIC COMMUNICATIONS

ISSN 2056-9890

Received 12 April 2018

Accepted 23 April 2018

Edited by D.-J. Xu, Zhejiang University (Yuquan Campus), China

Keywords: crystal structure; ionic liquids; pyridiniminium salt; hydrogen bonding; Hirshfeld surface analysis.

CCDC reference: 1839059

Supporting information: this article has supporting information at journals.iucr.org/e

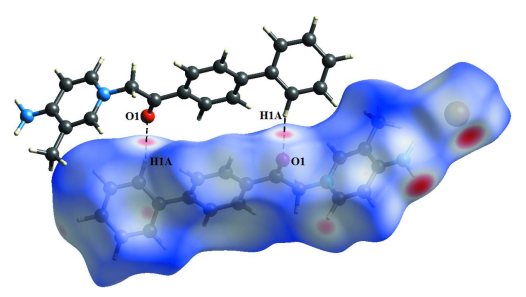

\section{Crystal structure and Hirshfeld surface analysis of a pyridiniminium bromide salt: 1-[2-([1,1'-biphenyl]- 4-yl)-2-oxoethyl]-3-methyl-1,4-dihydropyridin-4- iminium bromide}

S. N. Sheshadri, ${ }^{a}$ Huey Chong Kwong, ${ }^{b}$ C. S. Chidan Kumar, ${ }^{C} *$ Ching Kheng Quah, ${ }^{d}$ B. P. Siddaraju, ${ }^{\text {e }}$ M. K. Veeraiah, ${ }^{f}$ Muhammad Aiman Bin Abd Hamid ${ }^{d}$ and Ismail Warad $^{\text {g* }}$

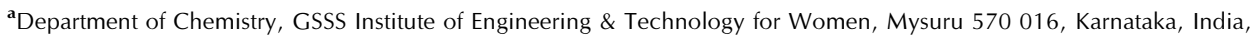
${ }^{\mathbf{b}}$ School of Chemical Sciences, Universiti Sains Malaysia, Penang 11800 USM, Malaysia, 'Department of Engineering Chemistry, Vidya Vikas Institute of Engineering \& Technology, Visvesvaraya Technological University, Alanahally, Mysuru 570 028, Karnataka, India, 'X-ray Crystallography Unit, School of Physics, Universiti Sains Malaysia, 11800 USM, Penang, Malaysia, ${ }^{\mathbf{e}}$ Department of Chemistry, Cauvery Institute of Technology, Mandya 571 402, Karnataka, India, ${ }^{\mathbf{f}}$ Department of Chemistry, Sri Siddhartha Institute of Technology, Tumkur 572 105, Karnataka, India, and ${ }^{\mathbf{g}}$ Department of Chemistry, Science College, An-Najah National University, PO Box 7, Nablus, West Bank, Palestinian Territories.

*Correspondence e-mail: chidankumar@gmail.com, khalil.i@najah.edu

In the cation of the title salt, $\mathrm{C}_{20} \mathrm{H}_{19} \mathrm{~N}_{2} \mathrm{O}^{+} \cdot \mathrm{Br}^{-}$, the phenyl rings are inclined to one another by $38.38(8)^{\circ}$, whereas the central phenyl ring and the pyridiniminium ring are almost perpendicular with a dihedral angle of $87.37(9)^{\circ}$. The $\mathrm{N}^{+}=\mathrm{C}$ cationic double bond was verified by the shortened bond length of 1.337 (2) $\AA$. In the crystal, the $\mathrm{Br}^{-}$anion is linked to the cation by an $\mathrm{N}-\mathrm{H} \cdots \mathrm{Br}$ hydrogen bond. $\mathrm{C}-\mathrm{H} \cdots \mathrm{O}$ hydrogen bonds link adjacent pyridiniminium cations into inversion dimers with an $R_{2}^{2}(18)$ graph-set motif. These dimers are stacked in a phenyl-phenyl T-shaped geometry through C$\mathrm{H} \cdots \pi$ interactions. A Hirshfeld surface analysis was conducted to verify the contributions of the different intermolecular interactions.

\section{Chemical context}

Over the past decade, ionic liquids have been the subject of intense research as a customizable replacement for volatile organic solvents because of their negligible vapor pressure, excellent thermal stability, high ionic conductivity and solvation ability (Davis, 2004). A wide range of applications using ionic liquids has been reported in many areas, such as their use as homogeneous and heterogeneous catalysts (Dong et al., 2016) and biological reaction media (Lopes et al., 2017), and in nuclear waste treatment (Ha et al., 2010) and water purification (Fuerhacker et al., 2012; Wang \& Wei, 2017).

In the view of the above and of our research interest in the synthesis of ionic liquids, we present in this study the crystal structure and Hirshfeld surface analysis of the title pyridiniminium halide salt.

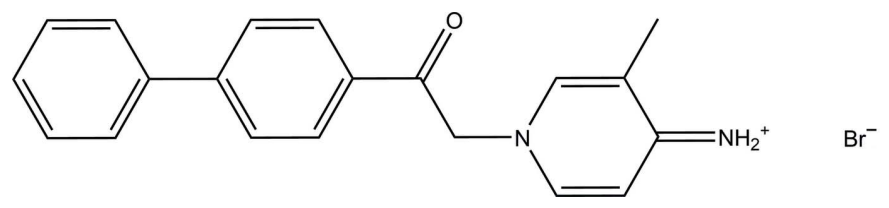




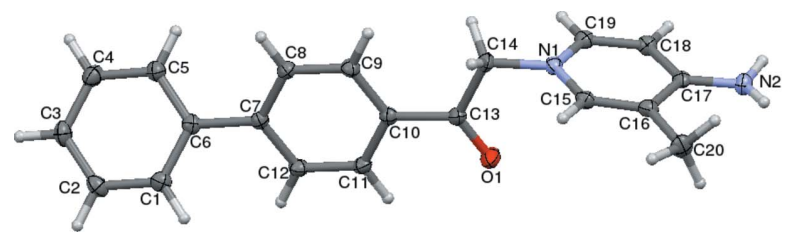

Figure 1

The molecular structure of the component ions of the title salt, indicating the atom-numbering scheme. Displacement ellipsoids are drawn at the $50 \%$ probability level.

\section{Structural commentary}

Fig. 1 shows the asymmetric unit of the title salt, consisting of one 1-(2-([1,1'-biphenyl]-4-yl)-2-oxoethyl)-3-methylpyridin$4(1 H)$-iminium cation and one bromide anion. The cation is constructed from a pyridiniminium ring $(\mathrm{N} 1 / \mathrm{C} 15-\mathrm{C} 19)$ and a biphenyl unit (C1-C6 and $\mathrm{C} 7-\mathrm{C} 12)$, interconnected by a $(\mathrm{C}=\mathrm{O})-\mathrm{C}$ ketone bridge. The biphenyl conformation experiences non-bounded steric repulsion between orthohydrogen atoms (Poater et al., 2006), with the phenyl rings inclined to one another by $38.38(8)^{\circ}$. The second phenyl ring $(\mathrm{C} 7-\mathrm{C} 12)$ is nearly parallel to the ketone bridge (O1/C13$\mathrm{C} 14)$, as shown by the torsion angles $\mathrm{C} 9-\mathrm{C} 10-\mathrm{C} 13-\mathrm{O} 1$ $\left[-179.10(18)^{\circ}\right]$ and $\mathrm{C} 9-\mathrm{C} 10-\mathrm{C} 13-\mathrm{C} 14\left[1.7(2)^{\circ}\right]$. Conversely, this phenyl ring is almost perpendicular to the pyridiniminium ring [dihedral angle $=87.37(9)^{\circ}$ ]. The bond lengths and angles in the cation are generally within normal ranges. However, the $\mathrm{N} 2-\mathrm{C} 17$ bond [1.337 (2) $\AA$ ] is shorter than expected for an $\mathrm{NH}_{2}-\mathrm{C}_{\mathrm{ar}}$ single bond [1.38 (3) $\AA$ ] although similar bond lengths have been observed in related compounds with an $\mathrm{N}^{+}=\mathrm{C}$ double bond (Chidan Kumar et al., 2017; Sharmila et al., 2014; Yue et al., 2013).
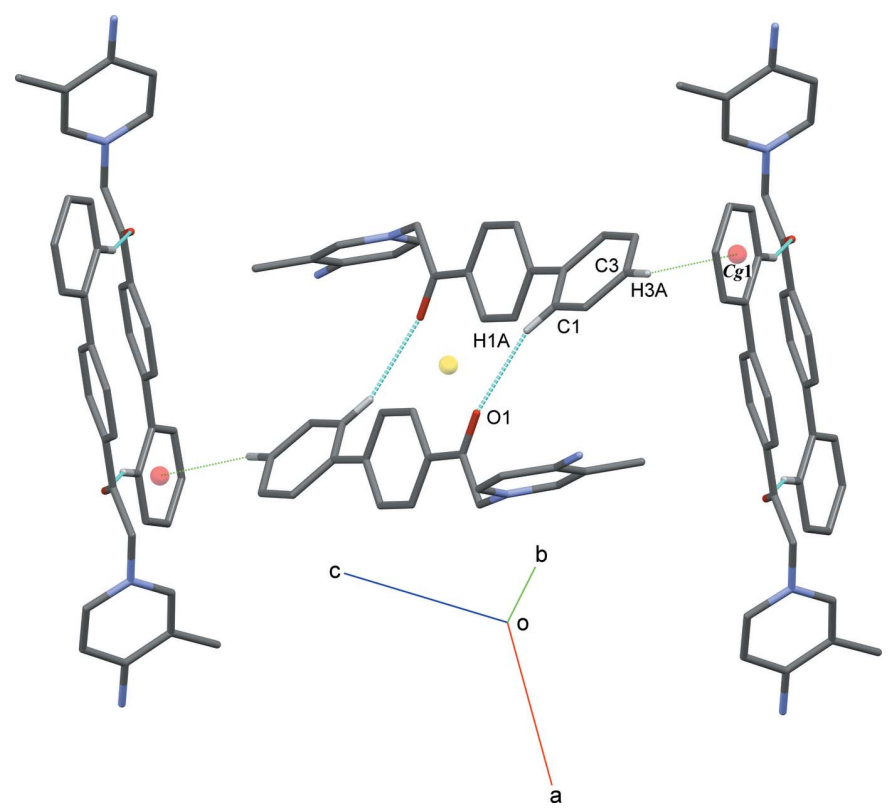

Figure 2

Partial packing diagram of the cation showing the $\mathrm{C} 1-\mathrm{H} 1 A \cdots \mathrm{O} 1$ hydrogen bonds (blue dashed lines) and $\mathrm{C} 3-\mathrm{H} 3 A \cdots \pi$ interactions (green dashed lines).
Table 1

Hydrogen-bond geometry $\left(\AA{ }^{\circ}\right)$.

$C g 1$ is the centroid of the $\mathrm{C} 1-\mathrm{C} 6$ ring.

\begin{tabular}{lllll}
\hline$D-\mathrm{H} \cdots A$ & $D-\mathrm{H}$ & $\mathrm{H} \cdots A$ & $D \cdots A$ & $D-\mathrm{H} \cdots A$ \\
\hline $\mathrm{N} 2-\mathrm{H} 1 N 2 \cdots \mathrm{Br} 1$ & $0.83(2)$ & $2.61(2)$ & $3.3514(17)$ & $150(2)$ \\
$\mathrm{N} 2-\mathrm{H} 2 N 2 \cdots \mathrm{Br} 1^{\mathrm{i}}$ & $0.86(2)$ & $2.52(2)$ & $3.3763(17)$ & $174(2)$ \\
$\mathrm{C} 1-\mathrm{H} 1 A \cdots \mathrm{O} 1^{\mathrm{ii}}$ & 0.95 & 2.52 & $3.431(2)$ & 162 \\
$\mathrm{C} 14-\mathrm{H} 14 A \cdots \mathrm{Br} 1^{\mathrm{iii}}$ & 0.99 & 2.88 & $3.710(2)$ & 141 \\
$\mathrm{C} 19-\mathrm{H} 19 A \cdots \mathrm{Br}^{\text {iv }}$ & 0.95 & 2.82 & $3.5415(18)$ & 134 \\
$\mathrm{C} 3-\mathrm{H} 3 A \cdots C g 1^{\mathrm{v}}$ & 0.95 & 2.81 & $3.6963(19)$ & 155 \\
\hline
\end{tabular}

Symmetry codes: (i) $-x+2,-y+3,-z+1$; (ii) $-x+1,-y+1,-z+1$; (iii) $-x+2,-y+2,-z+1$; (iv) $x,-y+\frac{5}{2}, z+\frac{1}{2}$; (v) $-x+1, y-\frac{1}{2},-z+\frac{3}{2}$.

\section{Supramolecular features}

In the crystal, the bromide anion is linked to the cation via an $\mathrm{N} 2-\mathrm{H} 2 N 2 \cdots \mathrm{Br} 1$ hydrogen bond (Table 1). The bromide anion is surrounded by three other cations with short $\mathrm{H} \cdots \mathrm{Br}$ contracts varying from 2.52 to $2.88 \AA$ (Table 1 ). Pairs of $\mathrm{C} 1-$ $\mathrm{H} 1 A \cdots \mathrm{O} 1$ hydrogen bonds link the pyridiniminium cations into inversion dimers with an $R_{2}^{2}(18)$ graph-set motif (Table 1 , Fig. 2). The dimers are stacked in a phenyl-phenyl T-shaped geometry through $\mathrm{C} 3-\mathrm{H} 3 A \cdots C g 1$ interactions $(C g 1$ is the centroid of the C1-C6 phenyl ring).

\section{Hirshfeld surface analysis}

The Hirshfeld surface analysis (Spackman \& Jayatilaka, 2009) of the title salt was generated by CrystalExplorer3.1 (Wolff et
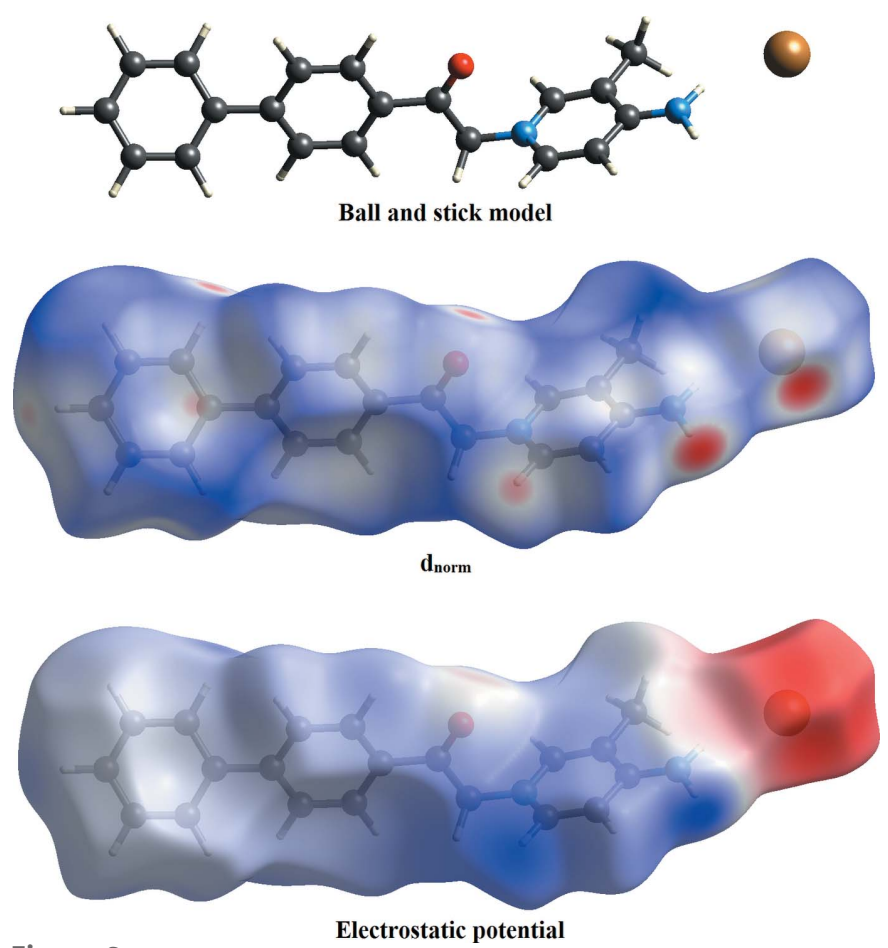

Figure 3

$d_{\text {norm }}$ and electrostatic potential mapped on Hirshfeld surfaces for visualizing the intermolecular contacts in the title salt. The ball-and-stick models shown here and in the following figures represent the different orientations corresponding to the Hirshfeld surfaces and their electrostatic potentials. 

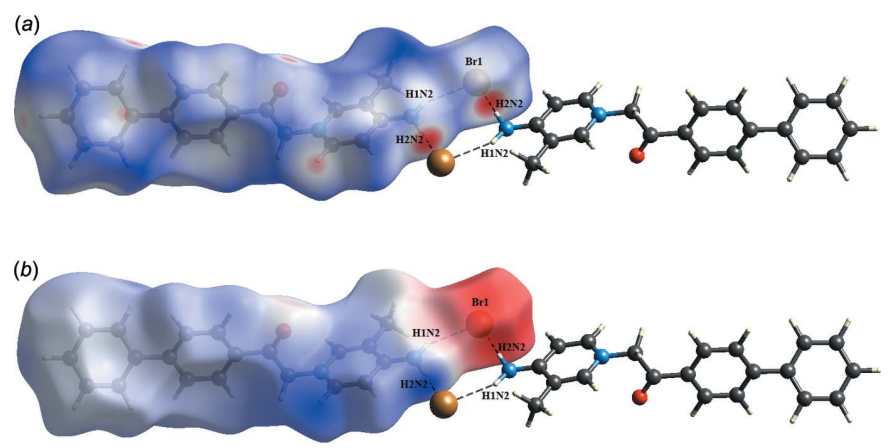

Figure 4

Visualization of $\mathrm{N}-\mathrm{H} \cdots \mathrm{O}$ hydrogen bond interactions through the $(a)$ $d_{\text {norm }}$ and $(b)$ electrostatic potential maps. Hydrogen bonds are represented by dashed lines.

al., 2012), and comprised $d_{\text {norm }}$ surface plots, electrostatic potentials and 2D fingerprint plots (Spackman \& McKinnon, 2002). The ball-and-stick model, $d_{\text {norm }}$ surface plots and electrostatic potentials of the title salt are shown in Fig. 3. Those plots were generated to quantify and visualize the intermolecular interactions and to explain the observed crystal packing. The dark-red spots on the $d_{\text {norm }}$ surface arise as a result of short interatomic contacts, while the other weak intermolecular interactions appear as light-red spots. Furthermore, negative electrostatic potential (red regions) in the electrostatic potential map indicates hydrogen-acceptor potential, whereas the hydrogen donors are represented by positive electrostatic potential (blue regions) (Spackman et al., 2008).

The $d_{\text {norm }}$ surface of the title salt shows a dark-red spot on the $\mathrm{N}-\mathrm{H}$ hydrogen atom and on the bromide atom, which is the result of the strong $\mathrm{N} 2-\mathrm{H} 1 N 2 \cdots \mathrm{Br} 1$ and $\mathrm{N} 2-$ $\mathrm{H} 2 \mathrm{~N} 2 \cdots \mathrm{Br} 1$ hydrogen bonds present in the structure (Fig. $4 a$ ). These observations are further confirmed by the respective electrostatic potential maps, where the atoms involved in strong hydrogen bonds are seen as dark-blue and dark-red regions (Fig. 4b). Beside those two short intermolecular contacts, the $\mathrm{C}-\mathrm{H} \cdots \mathrm{O}$ and $\mathrm{C}-\mathrm{H} \cdots \mathrm{Br}$ interactions are shown as light-red spots on the $d_{\text {norm }}$ surface (Fig. 5). Finally, the $\mathrm{C}-\mathrm{H} \cdots \pi$ interaction is shown as a light-red spot on the $d_{\text {norm }}$ surface (Fig. 6).

A quantitative analysis of the intermolecular interactions can be made by studying the fingerprint plots (FP). The FP is shown with characteristic pseudo-symmetry wings in the $d_{\mathrm{e}}$ and $d_{\mathrm{i}}$ diagonal axes represent the overall two-dimensional FP and those delineated into $\mathrm{H} \cdots \mathrm{H}, \mathrm{H} \cdots \mathrm{C} / \mathrm{C} \cdots \mathrm{H}, \mathrm{H} \cdots \mathrm{Br} /$ $\mathrm{Br} \cdots \mathrm{H}$ and $\mathrm{H} \cdots \mathrm{O} / \mathrm{O} \cdots \mathrm{H}$ contacts, respectively (Fig. 7). The most significant intermolecular interactions are the $\mathrm{H} \cdots \mathrm{H}$ interaction $(41.8 \%)$, which appear at the central region of the FP with $d_{\mathrm{e}}=d_{\mathrm{i}} \simeq 2.2 \AA$ (Fig. 7b). The reciprocal H $\cdots \mathrm{C} / \mathrm{C} \cdots \mathrm{H}$ interactions appear as two symmetrical broad wings with $d_{\mathrm{e}}+d_{\mathrm{i}} \simeq 2.7 \AA$ and contribute $29.2 \%$ to the Hirshfeld surface (Fig. 7c). The reciprocal $\mathrm{H} \cdots \mathrm{Br} / \mathrm{Br} \cdots \mathrm{H}$ and $\mathrm{H} \cdots \mathrm{O} / \mathrm{O} \cdots \mathrm{H}$ interactions with $16.7 \%$ and $7.3 \%$ contributions are present as sharp symmetrical spikes at diagonal axes $d_{\mathrm{e}}+d_{\mathrm{i}} \simeq 2.3$ and $2.4 \AA$ A , respectively (Fig. $7 d-e$ ). The percentage contributions (a)
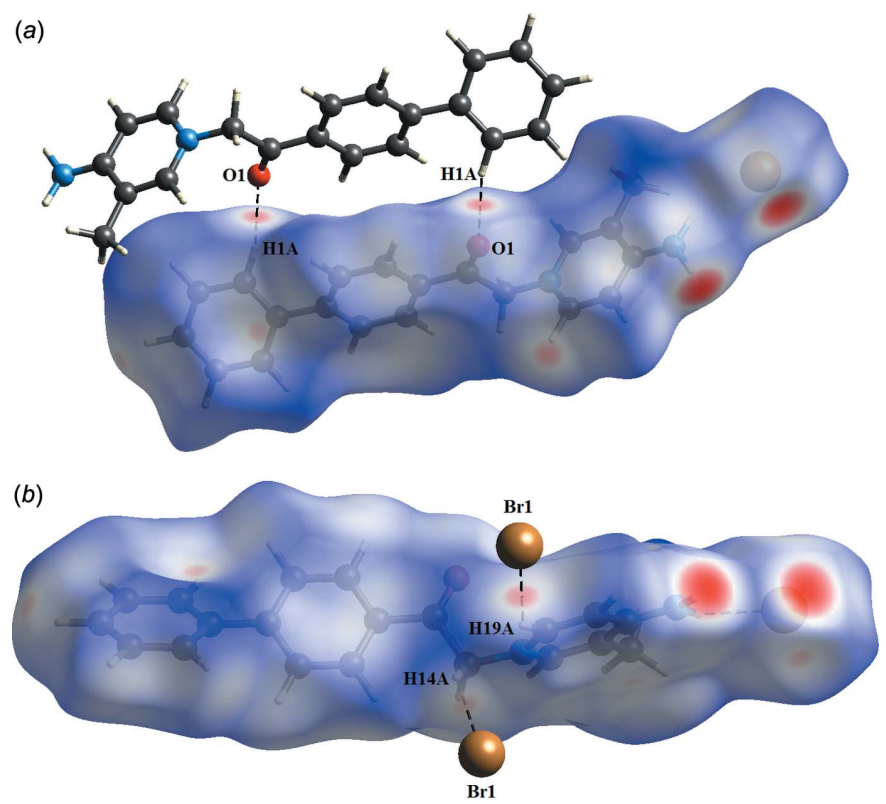

Figure 5

Visualization of (a) $\mathrm{C}-\mathrm{H} \cdots \mathrm{O}$ hydrogen bonds and $(b) \mathrm{C}-\mathrm{H} \cdots \mathrm{Br}$ interactions through the $d_{\text {norm }}$ maps. Hydrogen bonds are represented by dashed lines.

for other intermolecular contacts are less than $5 \%$ in the Hirshfeld surface mapping.

\section{Synthesis and crystallization}

The synthesis of the title compound is illustrated in Fig. 8. A mixture of 1-([1,1'-biphenyl]-4-yl)-2-bromoethan-1-one $(2.75 \mathrm{~g}, 10 \mathrm{mmol})$ and 3-methylpyridin-4-amine $(0.11 \mathrm{~g}$, $1 \mathrm{mmol}$ ) was dissolved in $10 \mathrm{ml}$ of toluene at room temperature, followed by stirring at $358 \mathrm{~K}$ for $18 \mathrm{~h}$. The completion of

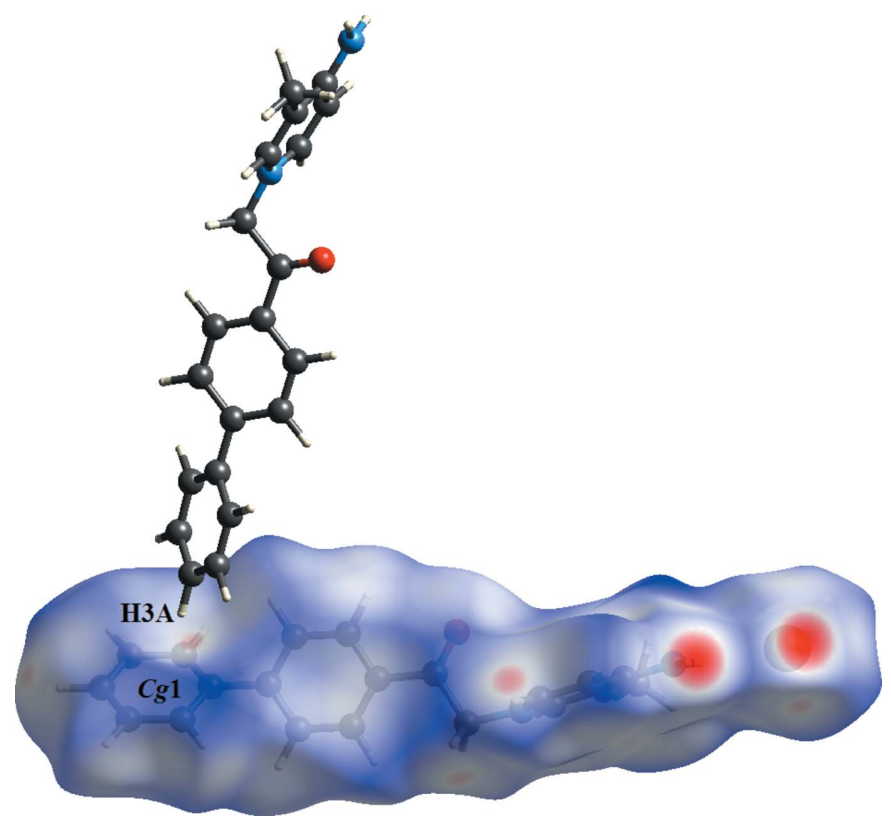

Figure 6

Visualization of $\mathrm{C}-\mathrm{H} \cdots \pi$ interactions through the $d_{\text {norm }}$ maps. 


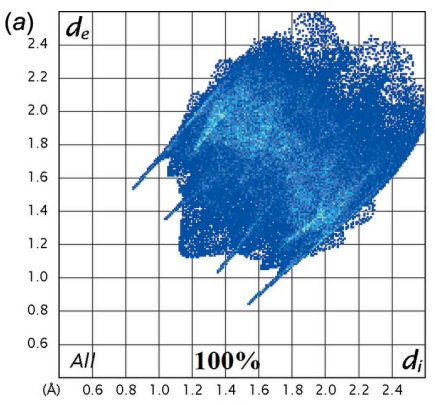

(b)
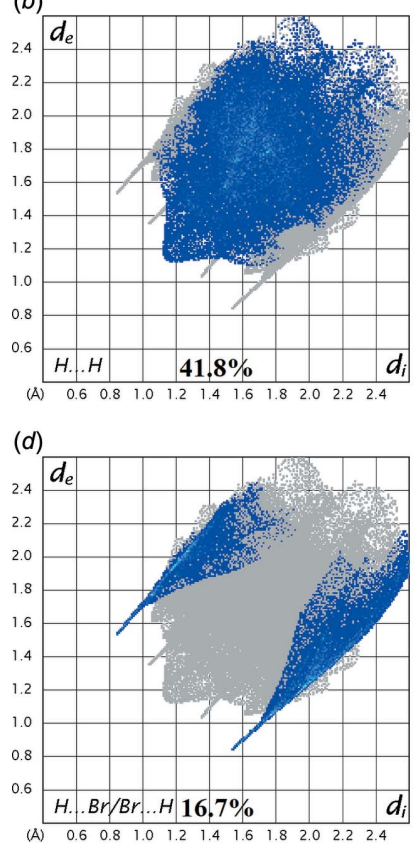

(c)

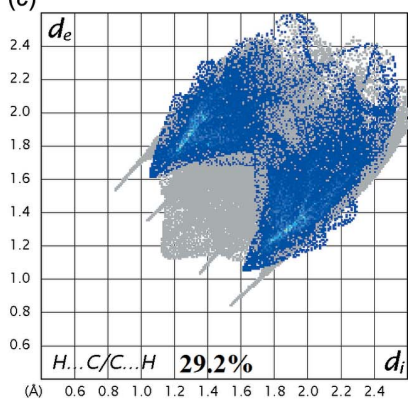

(e)

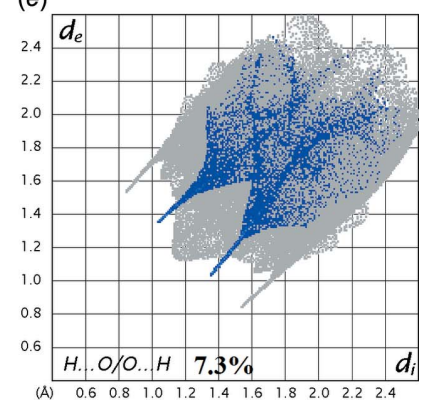

Figure 7

Two-dimensional fingerprint plots of the title salt showing the different percentage contributions for the various types of interactions.

the reaction was marked by the amount of the separated solid from the initially clear and homogenous mixture of the starting materials. The solid was filtered from the unreacted starting materials and solvent, and subsequently washed with ethyl acetate. The final pyridiniminium salt was obtained after the solid was dried under reduced pressure to remove all volatile organic compounds (Said et al., 2017). Plate-like yellow crystals were obtained by slow evaporation of a solution in acetone.

\section{Refinement}

Crystal data, data collection and structure refinement details are summarized in Table 2. C-bound $\mathrm{H}$ atoms were positioned

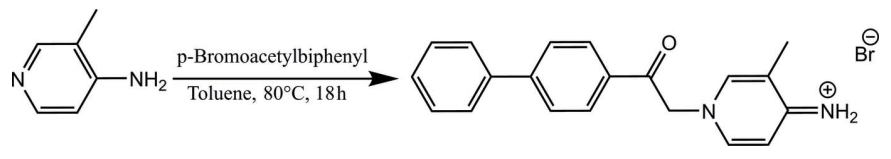

Figure 8

Synthesis of the title compound.

Table 2

Experimental details.

Crystal data

Chemical formula

$M_{\mathrm{r}}$

Crystal system, space group

Temperature (K)

$a, b, c(\AA)$

$\beta\left({ }^{\circ}\right)$

$V\left(\AA^{3}\right)$

$Z$

Radiation type

$\mu\left(\mathrm{mm}^{-1}\right)$

Crystal size (mm)

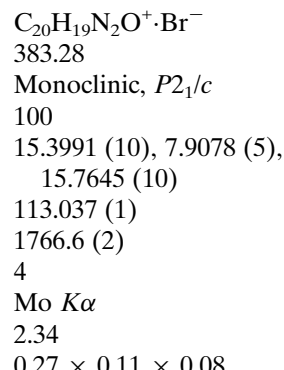

Data collection

Diffractometer

Absorption correction

$T_{\min }, T_{\max }$

No. of measured, independent and observed $[I>2 \sigma(I)]$ reflections

$R_{\text {int }}$

$(\sin \theta / \lambda)_{\max }\left(\AA^{-1}\right)$

Bruker APEXII DUO CCD area-
detector
Multi-scan (SADABS; Bruker,
2012)
$0.499,0.574$
$28949,4731,3913$
0.040
0.685

$0.030,0.075,1.03$
4731
226
H atoms treated by a mixture of
independent and constrained
refinement
$0.70,-0.23$

Refinement

$R\left[F^{2}>2 \sigma\left(F^{2}\right)\right], w R\left(F^{2}\right), S$

No. of reflections

No. of parameters

$\mathrm{H}$-atom treatment

$\Delta \rho_{\max }, \Delta \rho_{\min }\left(\mathrm{e} \AA^{-3}\right)$ $0.70,-0.23$

Computer programs: APEX2 and SAINT (Bruker, 2012), SHELXS97 (Sheldrick, 2008), SHELXL2013 (Sheldrick, 2015), Mercury (Macrae et al., 2006) and PLATON (Spek, 2009).

geometrically $[\mathrm{C}-\mathrm{H}=0.95-0.99 \AA]$ and refined using a riding model with $U_{\text {iso }}(\mathrm{H})=1.2$ or $1.5 U_{\text {eq }}(\mathrm{C})$. All $\mathrm{N}$-bound $\mathrm{H}$ atoms were located from a difference-Fourier map and freely refined.

\section{Acknowledgements}

HCK thanks the Malaysian Government for a MyBrain15 (MyPhD) scholarship.

\section{References}

Bruker (2012). APEX2, SAINT and SADABS. Bruker AXS Inc., Madison, Wisconsin, USA.

Chidan Kumar, C. S., Sim, A. J., Ng, W. Z., Chia, T. S., Loh, W.-S., Kwong, H. C., Quah, C. K., Naveen, S., Lokanath, N. K. \& Warad, I. (2017). Acta Cryst. E73, 927-931.

Davis, J. H. Jr (2004). Chem. Lett. 33, 1072-1077.

Dong, B., Song, H., Zhang, W., He, A. \& Yao, S. (2016). Curr. Org. Chem. 20, 2894-2910.

Fuerhacker, M., Haile, T. M., Kogelnig, D., Stojanovic, A. \& Keppler, B. (2012). Water Sci. Technol. 65, 1765-1773.

Ha, S. H., Menchavez, R. N. \& Koo, Y.-M. (2010). Korean J. Chem. Eng. 27, 1360-1365.

Lopes, J., Bermejo, M., Martín, Á. \& Cocero, M. (2017). ChemEngineering 1, 10; doi: 10.3390/chemengineering1020010

Macrae, C. F., Edgington, P. R., McCabe, P., Pidcock, E., Shields, G. P., Taylor, R., Towler, M. \& van de Streek, J. (2006). J. Appl. Cryst. 39, 453-457.

Poater, J., Solà, M. \& Bickelhaupt, F. M. (2006). Chem. Eur. J. 12, 2889-2895. 
Said, M. A., Aouad, M. R., Hughes, D. L., Almehmadi, M. A. \& Messali, M. (2017). Acta Cryst. E73, 1831-1834.

Sharmila, N., Sundar, T. V., Yasodha, A., Puratchikody, A. \& Sridhar, B. (2014). Acta Cryst. E70, o1293-o1294.

Sheldrick, G. M. (2008). Acta Cryst. A64, 112-122.

Sheldrick, G. M. (2015). Acta Cryst. C71, 3-8.

Spackman, M. A. \& Jayatilaka, D. (2009). CrystEngComm, 11, 19-32.

Spackman, M. A. \& McKinnon, J. J. (2002). CrystEngComm, 4, 378392.
Spackman, M. A., McKinnon, J. J. \& Jayatilaka, D. (2008). CrystEngComm, 10, 377-388.

Spek, A. L. (2009). Acta Cryst. D65, 148-155.

Wang, H. \& Wei, Y. (2017). RSC Adv. 7, 9079-9089.

Wolff, S. K., Grimwood, D. J., McKinnon, J. J., Turner, M. J., Jayatilaka, D. \& Spackman, M. A. (2012). University of Western Australia.

Yue, W.-W., Li, H.-J., Xiang, T., Qin, H., Sun, S.-D. \& Zhao, C.-S. (2013). J. Membr. Sci. 446, 79-91. 


\section{supporting information}

Acta Cryst. (2018). E74, 752-756 [https://doi.org/10.1107/S2056989018006217]

Crystal structure and Hirshfeld surface analysis of a pyridiniminium bromide salt: 1-[2-([1,1'-biphenyl]-4-yl)-2-oxoethyl]-3-methyl-1,4-dihydropyridin-4iminium bromide

\section{S. N. Sheshadri, Huey Chong Kwong, C. S. Chidan Kumar, Ching Kheng Quah, B. P. Siddaraju,} M. K. Veeraiah, Muhammad Aiman Bin Abd Hamid and Ismail Warad

Computing details

Data collection: APEX2 (Bruker, 2012); cell refinement: SAINT (Bruker, 2012); data reduction: SAINT (Bruker, 2012); program(s) used to solve structure: SHELXS97 (Sheldrick, 2008); program(s) used to refine structure: SHELXL2013

(Sheldrick, 2015); molecular graphics: SHELXL2013 (Sheldrick, 2015) and Mercury (Macrae et al., 2006); software used to prepare material for publication: SHELXL2013 (Sheldrick, 2015) and PLATON (Spek, 2009).

1-[2-([1,1'-Biphenyl]-4-yl)-2-oxoethyl]-3-methyl-1,4-dihydropyridin-4-iminium bromide

Crystal data

$\mathrm{C}_{20} \mathrm{H}_{19} \mathrm{~N}_{2} \mathrm{O}^{+} \cdot \mathrm{Br}^{-}$

$M_{r}=383.28$

Monoclinic, $P 2{ }_{1} / c$

$a=15.3991(10) \AA$

$b=7.9078(5) \AA$

$c=15.7645(10) \AA$

$\beta=113.037(1)^{\circ}$

$V=1766.6(2) \AA^{3}$

$Z=4$

Data collection

Bruker APEXII DUO CCD area-detector diffractometer

Radiation source: fine-focus sealed tube

Graphite monochromator

$\varphi$ and $\omega$ scans

Absorption correction: multi-scan

(SADABS; Bruker, 2012)

$T_{\text {min }}=0.499, T_{\max }=0.574$

Refinement

Refinement on $F^{2}$

Least-squares matrix: full

$R\left[F^{2}>2 \sigma\left(F^{2}\right)\right]=0.030$

$w R\left(F^{2}\right)=0.075$

$S=1.03$

4731 reflections
$F(000)=784$

$D_{\mathrm{x}}=1.441 \mathrm{Mg} \mathrm{m}^{-3}$

Mo $K \alpha$ radiation, $\lambda=0.71073 \AA$

Cell parameters from 7970 reflections

$\theta=2.6-28.7^{\circ}$

$\mu=2.34 \mathrm{~mm}^{-1}$

$T=100 \mathrm{~K}$

Plate, yellow

$0.27 \times 0.11 \times 0.08 \mathrm{~mm}$

28949 measured reflections

4731 independent reflections

3913 reflections with $I>2 \sigma(I)$

$R_{\text {int }}=0.040$

$\theta_{\text {max }}=29.2^{\circ}, \theta_{\min }=2.6^{\circ}$

$h=-20 \rightarrow 21$

$k=-10 \rightarrow 10$

$l=-21 \rightarrow 21$

226 parameters

0 restraints

Hydrogen site location: inferred from neighbouring sites

$\mathrm{H}$ atoms treated by a mixture of independent and constrained refinement 
$w=1 /\left[\sigma^{2}\left(F_{\mathrm{o}}^{2}\right)+(0.0375 P)^{2}+0.8608 P\right]$

where $P=\left(F_{\mathrm{o}}^{2}+2 F_{\mathrm{c}}^{2}\right) / 3$

$(\Delta / \sigma)_{\max }=0.001$

$$
\Delta \rho_{\max }=0.70 \text { e } \AA^{-3}
$$

\section{Special details}

Experimental. The following wavelength and cell were deduced by SADABS from the direction cosines etc. They are given here for emergency use only: CELL 0.71093 15.443 7.924 15.800 89.974 113.042 90.030

Geometry. All esds (except the esd in the dihedral angle between two 1.s. planes) are estimated using the full covariance matrix. The cell esds are taken into account individually in the estimation of esds in distances, angles and torsion angles; correlations between esds in cell parameters are only used when they are defined by crystal symmetry. An approximate (isotropic) treatment of cell esds is used for estimating esds involving l.s. planes.

Fractional atomic coordinates and isotropic or equivalent isotropic displacement parameters $\left(\AA^{2}\right)$

\begin{tabular}{|c|c|c|c|c|}
\hline & $x$ & $y$ & $z$ & $U_{\text {iso }} * / U_{\text {eq }}$ \\
\hline Br1 & $0.92732(2)$ & $1.53732(2)$ & $0.32391(2)$ & $0.02112(6)$ \\
\hline N1 & $0.85797(10)$ & $0.77203(18)$ & $0.52316(10)$ & $0.0165(3)$ \\
\hline N2 & $0.92877(11)$ & $1.25846(19)$ & $0.48455(12)$ & $0.0191(3)$ \\
\hline $\mathrm{H} 1 \mathrm{~N} 2$ & $0.9168(16)$ & $1.296(3)$ & $0.4320(17)$ & $0.028(6)^{*}$ \\
\hline $\mathrm{H} 2 \mathrm{~N} 2$ & $0.9689(16)$ & $1.310(3)$ & $0.5315(16)$ & $0.026(6)^{*}$ \\
\hline O1 & $0.68941(9)$ & $0.74507(17)$ & $0.53143(11)$ & 0.0288 \\
\hline $\mathrm{C} 1$ & $0.48304(13)$ & $-0.0147(2)$ & $0.60543(12)$ & $0.0190(3)$ \\
\hline $\mathrm{H} 1 \mathrm{~A}$ & 0.4442 & 0.0614 & 0.5597 & $0.023 *$ \\
\hline $\mathrm{C} 2$ & $0.44287(13)$ & $-0.1546(2)$ & $0.62926(12)$ & $0.0217(4)$ \\
\hline $\mathrm{H} 2 \mathrm{~A}$ & 0.3769 & -0.1739 & 0.5993 & $0.026 *$ \\
\hline $\mathrm{C} 3$ & $0.49803(13)$ & $-0.2662(2)$ & $0.69624(13)$ & $0.0232(4)$ \\
\hline $\mathrm{H} 3 \mathrm{~A}$ & 0.4703 & -0.3613 & 0.7127 & $0.028 *$ \\
\hline $\mathrm{C} 4$ & 0.59455 (14) & $-0.2375(2)$ & $0.73918(13)$ & $0.0241(4)$ \\
\hline $\mathrm{H} 4 \mathrm{~A}$ & 0.6328 & -0.3130 & 0.7856 & $0.029 *$ \\
\hline C5 & $0.63554(13)$ & $-0.0997(2)$ & $0.71483(13)$ & $0.0209(4)$ \\
\hline H5A & 0.7018 & -0.0830 & 0.7436 & $0.025^{*}$ \\
\hline C6 & $0.57967(12)$ & $0.0150(2)$ & $0.64790(12)$ & $0.0166(3)$ \\
\hline $\mathrm{C} 7$ & $0.62172(12)$ & $0.1671(2)$ & $0.62368(11)$ & 0.0160 \\
\hline $\mathrm{C} 8$ & $0.71004(12)$ & $0.1613(2)$ & $0.61812(12)$ & $0.0177(3)$ \\
\hline H8A & 0.7449 & 0.0587 & 0.6312 & $0.021 *$ \\
\hline C9 & $0.74715(12)$ & $0.3039(2)$ & $0.59364(12)$ & $0.0176(3)$ \\
\hline H9A & 0.8072 & 0.2981 & 0.5900 & $0.021 *$ \\
\hline $\mathrm{C} 10$ & $0.69697(12)$ & $0.4563(2)$ & $0.57416(12)$ & 0.0159 \\
\hline $\mathrm{C} 11$ & $0.60974(12)$ & $0.4633(2)$ & $0.58173(12)$ & 0.0173 \\
\hline H11A & 0.5756 & 0.5667 & 0.5703 & $0.021 *$ \\
\hline $\mathrm{C} 12$ & $0.57262(12)$ & $0.3209(2)$ & $0.60576(12)$ & $0.0181(3)$ \\
\hline $\mathrm{H} 12 \mathrm{~A}$ & 0.5129 & 0.3273 & 0.6102 & $0.022 *$ \\
\hline C13 & $0.73288(12)$ & $0.6126(2)$ & $0.54717(12)$ & $0.0174(3)$ \\
\hline C14 & $0.82786(12)$ & $0.6034(2)$ & $0.53803(13)$ & $0.0187(3)$ \\
\hline H14A & 0.8757 & 0.5539 & 0.5948 & $0.022 *$ \\
\hline H14B & 0.8224 & 0.5293 & 0.4856 & $0.022 *$ \\
\hline C15 & $0.83058(12)$ & $0.8351(2)$ & $0.43666(12)$ & 0.0178 \\
\hline $\mathrm{H} 15 \mathrm{~A}$ & 0.7958 & 0.7642 & 0.3861 & $0.021 *$ \\
\hline C16 & $0.85084(12)$ & $0.9961(2)$ & $0.41921(12)$ & $0.0181(3)$ \\
\hline
\end{tabular}




\begin{tabular}{lllll}
$\mathrm{C} 17$ & $0.90417(11)$ & $1.1006(2)$ & $0.49601(12)$ & $0.0160(3)$ \\
$\mathrm{C} 18$ & $0.92986(12)$ & $1.0325(2)$ & $0.58534(12)$ & $0.0168(3)$ \\
H18A & 0.9638 & 1.0999 & 0.6378 & $0.020^{*}$ \\
C19 & $0.90626(12)$ & $0.8711(2)$ & $0.59677(12)$ & $0.0175(3)$ \\
H19A & 0.9239 & 0.8270 & 0.6573 & $0.021^{*}$ \\
C20 & $0.81947(15)$ & $1.0614(3)$ & $0.32252(13)$ & $0.0268(4)$ \\
H20A & 0.7890 & 0.9700 & 0.2790 & $0.040^{*}$ \\
H20B & 0.8743 & 1.1026 & 0.3118 & $0.040^{*}$ \\
H20C & 0.7745 & 1.1541 & 0.3135 & $0.040^{*}$ \\
\hline
\end{tabular}

Atomic displacement parameters $\left(\AA^{2}\right)$

\begin{tabular}{lllllll}
\hline & $U^{11}$ & $U^{22}$ & $U^{33}$ & $U^{12}$ & $U^{13}$ & $U^{23}$ \\
\hline Br1 & $0.02487(10)$ & $0.02019(10)$ & $0.01683(9)$ & $-0.00208(7)$ & $0.00658(7)$ & $0.00115(7)$ \\
N1 & $0.0153(7)$ & $0.0152(7)$ & $0.0203(7)$ & $-0.0005(5)$ & $0.0083(6)$ & $-0.0004(6)$ \\
N2 & $0.0222(8)$ & $0.0166(7)$ & $0.0195(8)$ & $-0.0018(6)$ & $0.0091(6)$ & $0.0010(6)$ \\
O1 & $0.0230(7)$ & $0.0184(6)$ & $0.0487(9)$ & $0.0050(5)$ & $0.0179(6)$ & $0.0081(6)$ \\
C1 & $0.0190(8)$ & $0.0230(9)$ & $0.0155(8)$ & $-0.0001(7)$ & $0.0072(7)$ & $0.0000(7)$ \\
C2 & $0.0197(9)$ & $0.0271(9)$ & $0.0200(9)$ & $-0.0050(7)$ & $0.0097(7)$ & $-0.0031(7)$ \\
C3 & $0.0284(10)$ & $0.0218(9)$ & $0.0233(9)$ & $-0.0050(7)$ & $0.0144(8)$ & $0.0009(7)$ \\
C4 & $0.0272(10)$ & $0.0219(9)$ & $0.0245(9)$ & $0.0033(7)$ & $0.0116(8)$ & $0.0063(7)$ \\
C5 & $0.0197(8)$ & $0.0205(8)$ & $0.0230(9)$ & $0.0004(7)$ & $0.0090(7)$ & $0.0026(7)$ \\
C6 & $0.0193(8)$ & $0.0172(8)$ & $0.0155(8)$ & $-0.0003(6)$ & $0.0093(7)$ & $-0.0008(6)$ \\
C7 & $0.0165(8)$ & $0.0174(8)$ & $0.0134(8)$ & $-0.0011(6)$ & $0.0052(6)$ & $0.0001(6)$ \\
C8 & $0.0173(8)$ & $0.0158(8)$ & $0.0201(8)$ & $0.0011(6)$ & $0.0073(7)$ & $0.0006(6)$ \\
C9 & $0.0163(8)$ & $0.0178(8)$ & $0.0204(9)$ & $0.0007(6)$ & $0.0089(7)$ & $-0.0007(7)$ \\
C10 & $0.0160(8)$ & $0.0165(8)$ & $0.0145(8)$ & $-0.0005(6)$ & $0.0052(6)$ & $-0.0005(6)$ \\
C11 & $0.0161(8)$ & $0.0164(8)$ & $0.0181(8)$ & $0.0030(6)$ & $0.0053(6)$ & $0.0011(7)$ \\
C12 & $0.0147(8)$ & $0.0203(8)$ & $0.0198(8)$ & $0.0020(6)$ & $0.0072(7)$ & $0.0008(7)$ \\
C13 & $0.0151(8)$ & $0.0162(8)$ & $0.0198(8)$ & $0.0005(6)$ & $0.0054(7)$ & $0.0011(7)$ \\
C14 & $0.0189(8)$ & $0.0136(7)$ & $0.0248(9)$ & $0.0001(6)$ & $0.0096(7)$ & $0.0012(7)$ \\
C15 & $0.0166(8)$ & $0.0196(8)$ & $0.0164(8)$ & $-0.0010(6)$ & $0.0056(7)$ & $-0.0014(6)$ \\
C16 & $0.0145(8)$ & $0.0225(9)$ & $0.0171(8)$ & $0.0010(6)$ & $0.0059(7)$ & $0.0009(6)$ \\
C17 & $0.0141(8)$ & $0.0163(8)$ & $0.0199(8)$ & $0.0019(6)$ & $0.0092(7)$ & $-0.0007(7)$ \\
C18 & $0.0181(8)$ & $0.0183(8)$ & $0.0152(8)$ & $-0.0007(7)$ & $0.0078(6)$ & $-0.0023(7)$ \\
C19 & $0.0179(8)$ & $0.0189(8)$ & $0.0178(8)$ & $0.0024(6)$ & $0.0093(7)$ & $0.0016(7)$ \\
C20 & $0.0282(10)$ & $0.0311(10)$ & $0.0169(9)$ & $-0.0046(8)$ & $0.0044(7)$ & $0.0049(8)$ \\
& & & & & & \\
& & & & & & \\
& & & & & &
\end{tabular}

Geometric parameters $\left(A,{ }^{\circ}\right)$

\begin{tabular}{llll}
\hline $\mathrm{N} 1-\mathrm{C} 15$ & $1.355(2)$ & $\mathrm{C} 8-\mathrm{H} 8 \mathrm{~A}$ & 0.9500 \\
$\mathrm{~N} 1-\mathrm{C} 19$ & $1.356(2)$ & $\mathrm{C} 9-\mathrm{C} 10$ & $1.400(2)$ \\
$\mathrm{N} 1-\mathrm{C} 14$ & $1.460(2)$ & $\mathrm{C} 9-\mathrm{H} 9 \mathrm{~A}$ & 0.9500 \\
$\mathrm{~N} 2-\mathrm{C} 17$ & $1.337(2)$ & $\mathrm{C} 10-\mathrm{C} 11$ & $1.395(2)$ \\
$\mathrm{N} 2-\mathrm{H} 1 \mathrm{~N} 2$ & $0.83(2)$ & $\mathrm{C} 10-\mathrm{C} 13$ & $1.483(2)$ \\
$\mathrm{N} 2-\mathrm{H} 2 \mathrm{~N} 2$ & $0.86(2)$ & $\mathrm{C} 11-\mathrm{C} 12$ & $1.381(2)$ \\
$\mathrm{O} 1-\mathrm{C} 13$ & $1.215(2)$ & $\mathrm{C} 11-\mathrm{H} 11 \mathrm{~A}$ & 0.9500 \\
$\mathrm{C} 1-\mathrm{C} 2$ & $1.389(3)$ & $\mathrm{C} 12-\mathrm{H} 12 \mathrm{~A}$ & 0.9500
\end{tabular}




\begin{tabular}{|c|c|c|c|}
\hline $\mathrm{C} 1-\mathrm{C} 6$ & $1.392(2)$ & $\mathrm{C} 13-\mathrm{C} 14$ & $1.526(2)$ \\
\hline $\mathrm{C} 1-\mathrm{H} 1 \mathrm{~A}$ & 0.9500 & $\mathrm{C} 14-\mathrm{H} 14 \mathrm{~A}$ & 0.9900 \\
\hline $\mathrm{C} 2-\mathrm{C} 3$ & $1.383(3)$ & C14-H14B & 0.9900 \\
\hline $\mathrm{C} 2-\mathrm{H} 2 \mathrm{~A}$ & 0.9500 & $\mathrm{C} 15-\mathrm{C} 16$ & $1.365(2)$ \\
\hline $\mathrm{C} 3-\mathrm{C} 4$ & $1.390(3)$ & $\mathrm{C} 15-\mathrm{H} 15 \mathrm{~A}$ & 0.9500 \\
\hline $\mathrm{C} 3-\mathrm{H} 3 \mathrm{~A}$ & 0.9500 & $\mathrm{C} 16-\mathrm{C} 17$ & $1.430(2)$ \\
\hline $\mathrm{C} 4-\mathrm{C} 5$ & $1.387(3)$ & $\mathrm{C} 16-\mathrm{C} 20$ & $1.499(3)$ \\
\hline $\mathrm{C} 4-\mathrm{H} 4 \mathrm{~A}$ & 0.9500 & $\mathrm{C} 17-\mathrm{C} 18$ & $1.412(2)$ \\
\hline $\mathrm{C} 5-\mathrm{C} 6$ & $1.402(2)$ & $\mathrm{C} 18-\mathrm{C} 19$ & $1.358(2)$ \\
\hline $\mathrm{C} 5-\mathrm{H} 5 \mathrm{~A}$ & 0.9500 & $\mathrm{C} 18-\mathrm{H} 18 \mathrm{~A}$ & 0.9500 \\
\hline $\mathrm{C} 6-\mathrm{C} 7$ & $1.485(2)$ & $\mathrm{C} 19-\mathrm{H} 19 \mathrm{~A}$ & 0.9500 \\
\hline $\mathrm{C} 7-\mathrm{C} 8$ & $1.397(2)$ & $\mathrm{C} 20-\mathrm{H} 20 \mathrm{~A}$ & 0.9800 \\
\hline $\mathrm{C} 7-\mathrm{C} 12$ & $1.401(2)$ & $\mathrm{C} 20-\mathrm{H} 20 \mathrm{~B}$ & 0.9800 \\
\hline $\mathrm{C} 8-\mathrm{C} 9$ & $1.385(2)$ & $\mathrm{C} 20-\mathrm{H} 20 \mathrm{C}$ & 0.9800 \\
\hline $\mathrm{C} 15-\mathrm{N} 1-\mathrm{C} 19$ & $119.94(15)$ & $\mathrm{C} 12-\mathrm{C} 11-\mathrm{C} 10$ & $120.46(16)$ \\
\hline $\mathrm{C} 15-\mathrm{N} 1-\mathrm{C} 14$ & $120.28(15)$ & $\mathrm{C} 12-\mathrm{C} 11-\mathrm{H} 11 \mathrm{~A}$ & 119.8 \\
\hline $\mathrm{C} 19-\mathrm{N} 1-\mathrm{C} 14$ & $119.51(15)$ & $\mathrm{C} 10-\mathrm{C} 11-\mathrm{H} 11 \mathrm{~A}$ & 119.8 \\
\hline $\mathrm{C} 17-\mathrm{N} 2-\mathrm{H} 1 \mathrm{~N} 2$ & $120.3(16)$ & $\mathrm{C} 11-\mathrm{C} 12-\mathrm{C} 7$ & $120.95(16)$ \\
\hline $\mathrm{C} 17-\mathrm{N} 2-\mathrm{H} 2 \mathrm{~N} 2$ & $118.3(15)$ & $\mathrm{C} 11-\mathrm{C} 12-\mathrm{H} 12 \mathrm{~A}$ & 119.5 \\
\hline $\mathrm{H} 1 \mathrm{~N} 2-\mathrm{N} 2-\mathrm{H} 2 \mathrm{~N} 2$ & $120(2)$ & $\mathrm{C} 7-\mathrm{C} 12-\mathrm{H} 12 \mathrm{~A}$ & 119.5 \\
\hline $\mathrm{C} 2-\mathrm{C} 1-\mathrm{C} 6$ & $120.64(17)$ & $\mathrm{O} 1-\mathrm{C} 13-\mathrm{C} 10$ & $122.65(16)$ \\
\hline $\mathrm{C} 2-\mathrm{C} 1-\mathrm{H} 1 \mathrm{~A}$ & 119.7 & $\mathrm{O} 1-\mathrm{C} 13-\mathrm{C} 14$ & $119.56(16)$ \\
\hline $\mathrm{C} 6-\mathrm{C} 1-\mathrm{H} 1 \mathrm{~A}$ & 119.7 & $\mathrm{C} 10-\mathrm{C} 13-\mathrm{C} 14$ & $117.78(15)$ \\
\hline $\mathrm{C} 3-\mathrm{C} 2-\mathrm{C} 1$ & $120.60(17)$ & $\mathrm{N} 1-\mathrm{C} 14-\mathrm{C} 13$ & $110.35(14)$ \\
\hline $\mathrm{C} 3-\mathrm{C} 2-\mathrm{H} 2 \mathrm{~A}$ & 119.7 & $\mathrm{~N} 1-\mathrm{C} 14-\mathrm{H} 14 \mathrm{~A}$ & 109.6 \\
\hline $\mathrm{C} 1-\mathrm{C} 2-\mathrm{H} 2 \mathrm{~A}$ & 119.7 & $\mathrm{C} 13-\mathrm{C} 14-\mathrm{H} 14 \mathrm{~A}$ & 109.6 \\
\hline $\mathrm{C} 2-\mathrm{C} 3-\mathrm{C} 4$ & $119.21(17)$ & $\mathrm{N} 1-\mathrm{C} 14-\mathrm{H} 14 \mathrm{~B}$ & 109.6 \\
\hline $\mathrm{C} 2-\mathrm{C} 3-\mathrm{H} 3 \mathrm{~A}$ & 120.4 & $\mathrm{C} 13-\mathrm{C} 14-\mathrm{H} 14 \mathrm{~B}$ & 109.6 \\
\hline $\mathrm{C} 4-\mathrm{C} 3-\mathrm{H} 3 \mathrm{~A}$ & 120.4 & $\mathrm{H} 14 \mathrm{~A}-\mathrm{C} 14-\mathrm{H} 14 \mathrm{~B}$ & 108.1 \\
\hline $\mathrm{C} 5-\mathrm{C} 4-\mathrm{C} 3$ & $120.62(17)$ & $\mathrm{N} 1-\mathrm{C} 15-\mathrm{C} 16$ & $122.72(16)$ \\
\hline $\mathrm{C} 5-\mathrm{C} 4-\mathrm{H} 4 \mathrm{~A}$ & 119.7 & $\mathrm{~N} 1-\mathrm{C} 15-\mathrm{H} 15 \mathrm{~A}$ & 118.6 \\
\hline $\mathrm{C} 3-\mathrm{C} 4-\mathrm{H} 4 \mathrm{~A}$ & 119.7 & $\mathrm{C} 16-\mathrm{C} 15-\mathrm{H} 15 \mathrm{~A}$ & 118.6 \\
\hline $\mathrm{C} 4-\mathrm{C} 5-\mathrm{C} 6$ & $120.33(17)$ & $\mathrm{C} 15-\mathrm{C} 16-\mathrm{C} 17$ & $118.01(16)$ \\
\hline $\mathrm{C} 4-\mathrm{C} 5-\mathrm{H} 5 \mathrm{~A}$ & 119.8 & $\mathrm{C} 15-\mathrm{C} 16-\mathrm{C} 20$ & $121.21(17)$ \\
\hline $\mathrm{C} 6-\mathrm{C} 5-\mathrm{H} 5 \mathrm{~A}$ & 119.8 & $\mathrm{C} 17-\mathrm{C} 16-\mathrm{C} 20$ & $120.78(16)$ \\
\hline $\mathrm{C} 1-\mathrm{C} 6-\mathrm{C} 5$ & $118.57(16)$ & $\mathrm{N} 2-\mathrm{C} 17-\mathrm{C} 18$ & $120.51(16)$ \\
\hline $\mathrm{C} 1-\mathrm{C} 6-\mathrm{C} 7$ & $120.31(16)$ & $\mathrm{N} 2-\mathrm{C} 17-\mathrm{C} 16$ & $121.62(16)$ \\
\hline $\mathrm{C} 5-\mathrm{C} 6-\mathrm{C} 7$ & $121.11(16)$ & $\mathrm{C} 18-\mathrm{C} 17-\mathrm{C} 16$ & $117.87(16)$ \\
\hline $\mathrm{C} 8-\mathrm{C} 7-\mathrm{C} 12$ & $118.53(16)$ & $\mathrm{C} 19-\mathrm{C} 18-\mathrm{C} 17$ & $120.40(16)$ \\
\hline $\mathrm{C} 8-\mathrm{C} 7-\mathrm{C} 6$ & $121.51(15)$ & $\mathrm{C} 19-\mathrm{C} 18-\mathrm{H} 18 \mathrm{~A}$ & 119.8 \\
\hline $\mathrm{C} 12-\mathrm{C} 7-\mathrm{C} 6$ & $119.96(15)$ & $\mathrm{C} 17-\mathrm{C} 18-\mathrm{H} 18 \mathrm{~A}$ & 119.8 \\
\hline $\mathrm{C} 9-\mathrm{C} 8-\mathrm{C} 7$ & $120.55(16)$ & $\mathrm{N} 1-\mathrm{C} 19-\mathrm{C} 18$ & $121.01(16)$ \\
\hline $\mathrm{C} 9-\mathrm{C} 8-\mathrm{H} 8 \mathrm{~A}$ & 119.7 & $\mathrm{~N} 1-\mathrm{C} 19-\mathrm{H} 19 \mathrm{~A}$ & 119.5 \\
\hline $\mathrm{C} 7-\mathrm{C} 8-\mathrm{H} 8 \mathrm{~A}$ & 119.7 & $\mathrm{C} 18-\mathrm{C} 19-\mathrm{H} 19 \mathrm{~A}$ & 119.5 \\
\hline $\mathrm{C} 8-\mathrm{C} 9-\mathrm{C} 10$ & $120.64(16)$ & $\mathrm{C} 16-\mathrm{C} 20-\mathrm{H} 20 \mathrm{~A}$ & 109.5 \\
\hline $\mathrm{C} 8-\mathrm{C} 9-\mathrm{H} 9 \mathrm{~A}$ & 119.7 & $\mathrm{C} 16-\mathrm{C} 20-\mathrm{H} 20 \mathrm{~B}$ & 109.5 \\
\hline $\mathrm{C} 10-\mathrm{C} 9-\mathrm{H} 9 \mathrm{~A}$ & 119.7 & $\mathrm{H} 20 \mathrm{~A}-\mathrm{C} 20-\mathrm{H} 20 \mathrm{~B}$ & 109.5 \\
\hline
\end{tabular}




\begin{tabular}{|c|c|c|c|}
\hline $\mathrm{C} 11-\mathrm{C} 10-\mathrm{C} 9$ & $118.85(16)$ & $\mathrm{C} 16-\mathrm{C} 20-\mathrm{H} 20 \mathrm{C}$ & 109.5 \\
\hline $\mathrm{C} 11-\mathrm{C} 10-\mathrm{C} 13$ & $118.12(15)$ & $\mathrm{H} 20 \mathrm{~A}-\mathrm{C} 20-\mathrm{H} 20 \mathrm{C}$ & 109.5 \\
\hline $\mathrm{C} 9-\mathrm{C} 10-\mathrm{C} 13$ & $123.02(16)$ & $\mathrm{H} 20 \mathrm{~B}-\mathrm{C} 20-\mathrm{H} 20 \mathrm{C}$ & 109.5 \\
\hline $\mathrm{C} 6-\mathrm{C} 1-\mathrm{C} 2-\mathrm{C} 3$ & $0.6(3)$ & $\mathrm{C} 11-\mathrm{C} 10-\mathrm{C} 13-\mathrm{O} 1$ & $-0.1(3)$ \\
\hline $\mathrm{C} 1-\mathrm{C} 2-\mathrm{C} 3-\mathrm{C} 4$ & $-0.5(3)$ & $\mathrm{C} 9-\mathrm{C} 10-\mathrm{C} 13-\mathrm{O} 1$ & $-179.10(18)$ \\
\hline $\mathrm{C} 2-\mathrm{C} 3-\mathrm{C} 4-\mathrm{C} 5$ & $-0.6(3)$ & $\mathrm{C} 11-\mathrm{C} 10-\mathrm{C} 13-\mathrm{C} 14$ & $-179.35(16)$ \\
\hline $\mathrm{C} 3-\mathrm{C} 4-\mathrm{C} 5-\mathrm{C} 6$ & $1.5(3)$ & $\mathrm{C} 9-\mathrm{C} 10-\mathrm{C} 13-\mathrm{C} 14$ & $1.7(2)$ \\
\hline $\mathrm{C} 2-\mathrm{C} 1-\mathrm{C} 6-\mathrm{C} 5$ & $0.3(3)$ & $\mathrm{C} 15-\mathrm{N} 1-\mathrm{C} 14-\mathrm{C} 13$ & $-87.86(19)$ \\
\hline $\mathrm{C} 2-\mathrm{C} 1-\mathrm{C} 6-\mathrm{C} 7$ & $-178.51(16)$ & $\mathrm{C} 19-\mathrm{N} 1-\mathrm{C} 14-\mathrm{C} 13$ & $86.16(18)$ \\
\hline $\mathrm{C} 4-\mathrm{C} 5-\mathrm{C} 6-\mathrm{C} 1$ & $-1.4(3)$ & $\mathrm{O} 1-\mathrm{C} 13-\mathrm{C} 14-\mathrm{N} 1$ & $8.2(2)$ \\
\hline $\mathrm{C} 4-\mathrm{C} 5-\mathrm{C} 6-\mathrm{C} 7$ & $177.47(17)$ & $\mathrm{C} 10-\mathrm{C} 13-\mathrm{C} 14-\mathrm{N} 1$ & $-172.54(14)$ \\
\hline $\mathrm{C} 1-\mathrm{C} 6-\mathrm{C} 7-\mathrm{C} 8$ & $-142.49(18)$ & $\mathrm{C} 19-\mathrm{N} 1-\mathrm{C} 15-\mathrm{C} 16$ & $1.1(3)$ \\
\hline $\mathrm{C} 5-\mathrm{C} 6-\mathrm{C} 7-\mathrm{C} 8$ & $38.7(3)$ & $\mathrm{C} 14-\mathrm{N} 1-\mathrm{C} 15-\mathrm{C} 16$ & $175.11(16)$ \\
\hline $\mathrm{C} 1-\mathrm{C} 6-\mathrm{C} 7-\mathrm{C} 12$ & $37.5(2)$ & $\mathrm{N} 1-\mathrm{C} 15-\mathrm{C} 16-\mathrm{C} 17$ & $0.7(3)$ \\
\hline $\mathrm{C} 5-\mathrm{C} 6-\mathrm{C} 7-\mathrm{C} 12$ & $-141.33(18)$ & $\mathrm{N} 1-\mathrm{C} 15-\mathrm{C} 16-\mathrm{C} 20$ & $179.90(17)$ \\
\hline $\mathrm{C} 12-\mathrm{C} 7-\mathrm{C} 8-\mathrm{C} 9$ & $-1.2(3)$ & $\mathrm{C} 15-\mathrm{C} 16-\mathrm{C} 17-\mathrm{N} 2$ & $178.41(16)$ \\
\hline $\mathrm{C} 6-\mathrm{C} 7-\mathrm{C} 8-\mathrm{C} 9$ & $178.73(16)$ & $\mathrm{C} 20-\mathrm{C} 16-\mathrm{C} 17-\mathrm{N} 2$ & $-0.8(3)$ \\
\hline $\mathrm{C} 7-\mathrm{C} 8-\mathrm{C} 9-\mathrm{C} 10$ & $0.1(3)$ & $\mathrm{C} 15-\mathrm{C} 16-\mathrm{C} 17-\mathrm{C} 18$ & $-2.1(2)$ \\
\hline $\mathrm{C} 8-\mathrm{C} 9-\mathrm{C} 10-\mathrm{C} 11$ & $1.4(3)$ & $\mathrm{C} 20-\mathrm{C} 16-\mathrm{C} 17-\mathrm{C} 18$ & $178.76(17)$ \\
\hline $\mathrm{C} 8-\mathrm{C} 9-\mathrm{C} 10-\mathrm{C} 13$ & $-179.68(16)$ & $\mathrm{N} 2-\mathrm{C} 17-\mathrm{C} 18-\mathrm{C} 19$ & $-178.81(16)$ \\
\hline $\mathrm{C} 9-\mathrm{C} 10-\mathrm{C} 11-\mathrm{C} 12$ & $-1.6(3)$ & $\mathrm{C} 16-\mathrm{C} 17-\mathrm{C} 18-\mathrm{C} 19$ & $1.7(2)$ \\
\hline $\mathrm{C} 13-\mathrm{C} 10-\mathrm{C} 11-\mathrm{C} 12$ & $179.36(16)$ & $\mathrm{C} 15-\mathrm{N} 1-\mathrm{C} 19-\mathrm{C} 18$ & $-1.6(2)$ \\
\hline $\mathrm{C} 10-\mathrm{C} 11-\mathrm{C} 12-\mathrm{C} 7$ & $0.5(3)$ & $\mathrm{C} 14-\mathrm{N} 1-\mathrm{C} 19-\mathrm{C} 18$ & $-175.62(15)$ \\
\hline $\mathrm{C} 8-\mathrm{C} 7-\mathrm{C} 12-\mathrm{C} 11$ & $1.0(3)$ & $\mathrm{C} 17-\mathrm{C} 18-\mathrm{C} 19-\mathrm{N} 1$ & $0.2(3)$ \\
\hline $\mathrm{C} 6-\mathrm{C} 7-\mathrm{C} 12-\mathrm{C} 11$ & $-179.00(16)$ & & \\
\hline
\end{tabular}

Hydrogen-bond geometry $\left(A,{ }^{\circ}\right)$

$\mathrm{Cg} 1$ is the centroid of the $\mathrm{C} 1-\mathrm{C} 6$ ring.

\begin{tabular}{lllll}
\hline$D-\mathrm{H} \cdots A$ & $D-\mathrm{H}$ & $\mathrm{H} \cdots A$ & $D \cdots A$ & $D-\mathrm{H} \cdots A$ \\
\hline $\mathrm{N} 2-\mathrm{H} 1 N 2 \cdots \mathrm{Br} 1$ & $0.83(2)$ & $2.61(2)$ & $3.3514(17)$ & $150(2)$ \\
$\mathrm{N} 2-\mathrm{H} 2 N 2 \cdots \mathrm{Br} 1^{\mathrm{i}}$ & $0.86(2)$ & $2.52(2)$ & $3.3763(17)$ & $174(2)$ \\
$\mathrm{C} 1-\mathrm{H} 1 A \cdots \mathrm{O} 1^{\mathrm{ii}}$ & 0.95 & 2.52 & $3.431(2)$ & 162 \\
$\mathrm{C} 14-\mathrm{H} 14 A \cdots \mathrm{Br} 1^{\mathrm{iii}}$ & 0.99 & 2.88 & $3.710(2)$ & 141 \\
$\mathrm{C} 19-\mathrm{H} 19 A \cdots \mathrm{Br} 1^{\mathrm{iv}}$ & 0.95 & 2.82 & $3.5415(18)$ & 134 \\
$\mathrm{C} 3-\mathrm{H} 3 A \cdots C g 1^{\mathrm{v}}$ & 0.95 & 2.81 & $3.6963(19)$ & 155
\end{tabular}

Symmetry codes: (i) $-x+2,-y+3,-z+1$; (ii) $-x+1,-y+1,-z+1$; (iii) $-x+2,-y+2,-z+1$; (iv) $x,-y+5 / 2, z+1 / 2$; (v) $-x+1, y-1 / 2,-z+3 / 2$. 